\title{
Thermo-luminescence Response of Carbon Nanotubes and Some Other Familiar TL Materials Using Medical LINAC
}

\author{
N. A. Sohan ${ }^{1}$, M. J. Dewan ${ }^{1}$, A. K. M. M. Rahman', M. Al-Mamun ${ }^{2}$, M. I. Hosan ${ }^{1 *}$ \\ ${ }^{1}$ Department of Nuclear Engineering, University of Dhaka, Dhaka-1000, Bangladesh \\ ${ }^{2}$ Bangladesh Atomic Energy Commission, Dhaka-1000, Bangladesh
}

Received 5 February 2020, accepted in final revised form 11 June 2020

\begin{abstract}
Strain and impurity defects in carbon nanotubes (CNTs) particularly their potentiality as a new TL material has been studied over the years. In this research, our main objective is to explore the suitability of using CNTs and its composites in the area of TL dosimeter. For this purpose, a study was carried out between the TL responses of the dosimeters TLD-100, TLD-7000 and NaI-LiF pellets. To carry out this research, equivalent irradiations were performed with these pellets using clinical linear accelerator (LINAC) under $6 \mathrm{MV} \mathrm{X}$-ray photon beam. The dose range was from 0.5 to $5 \mathrm{~Gy}$. During irradiation, the dose rate was kept constant at $300 \mathrm{MU} / \mathrm{min}$. TLD reader was used to readout the samples in a flowing $\mathrm{N}_{2}$ atmosphere to reduce surface oxidation.During readout, pre-heat temperature was set initially at $50{ }^{\circ} \mathrm{C}$, acquired temperature rate $10{ }^{\circ} \mathrm{C} / \mathrm{s}$ and maximum annealed temperature was $300{ }^{\circ} \mathrm{C}$. Response of TLD-100 under varying dose was typically linear for any doses but other dosimeters TLD-7000 showed supra-linearity beyond 2 Gy and NaI-LiF pellets showed sub-linearity response after $2 \mathrm{~Gy}$. The TL glow peak of CNTs indicated that it was lying somewhere away from $300{ }^{\circ} \mathrm{C}$.
\end{abstract}

Keywords: CNT; TLD; LINAC; Water phantom; Supra-linearity; Sub-linearity.

(C) 2020 JSR Publications. ISSN: 2070-0237 (Print); 2070-0245 (Online). All rights reserved. doi: http://dx.doi.org/10.3329/jsr.v12i4.45336 J. Sci. Res. 12 (4), 455-462 (2020)

\section{Introduction}

Thermo-luminescent dosimeter (TLD) is one of the most popularly used and cost effective techniques for radiation dosimetry in the routine monitoring of occupational radiation exposure. It was invented in 1954 by Farrington Daniels of the University of WisconsinMadison [1]. Dosimetry deals with the measurement, calculation and assessment of directly or indirectly ionizing radiation dose absorbed by an object, usually the human body [2]. Chen et al. reported [3] that the traffic of charge carriers in a luminescent material during its excitation by irradiation and during readout either in the measurement of thermo-luminescence (TL) or optically stimulated luminescence (OSL) is governed by sets of coupled nonlinear differential equations. The analytical solution of these sets is

\footnotetext{
*Corresponding author: iqbalhosan@du.ac.bd
} 
usually not possible, and one can resort one of two options. Some researchers preferred to make simplifying assumptions and thus got approximate solutions whereas others performed simulations by solving the simultaneous equations numerically. Each of these routes has its pitfalls. Repeating the simulations with several sets of the physically plausible parameters would add credibility to the conclusions drawn. Horowitz observed [4] that the state-of-the-art in the use of thermo-luminescence for the measurement of energy imparted by ionizing radiation. Emphasis is on the advantages obtainable by the use of computerized glow curve analysis in (i) quality control, (ii) low dose environmental dosimetry, (iii) medical applications precision and micro-dosimetric applications, and (iv) mixed field ionization-density dosimetry. Possible frontiers of future research are highlighted: (i) vector representation in glow curve analysis, (ii) combined OSL/TL measurements, (iii) detection of sub-ionization electrons, (iv) requirements for new TL materials and (v) theoretical subjects involving kinetic modeling invoking localized/delocalized recombination applied to dose response and track structure theory including creation of defects. Hasan et al. [5] evaluated the dosimetric properties focusing on linearity and reproducibility of TLD 100 and TLD 7000.

It has been observed that using beta radiation delivering dose levels of a few gray (Gy) strain and impurity defects in carbon nanotubes give rise to significant TL yields. It provides an initial measurement of the extent to which electron trapping centers exist in various qualities of CNT from super-pure to raw. CNTs also have an effective atomic number similar to that of adipose tissue, making them suitable for soft tissue dosimetry [6, 7]. TLDs work by storing the energy they receive from the ionizing radiation until they are heated to high temperature. On heating, the absorbed energy is released in the form of visible light [8]. A plot of light intensity emitted against temperature is known as a glow curve. It contains several peaks, with each peak representing an intensity level. The interaction process between radiation and TLDs occurs in irradiation and thermolumiscence stages [9]. The performance of dosimeters is assessed through the study of characteristics such as linearity, dose response, energy response, stability, effect of the environment on dosimeter performance and others. The main objectives of this research were to carry out a comparison between the TL responses of the prepared dosimeters and TLD-100, determining the output stability of the proposed TLD and also the relative energy response of the TLD materials experimentally and compare with the theoretical values. During the experiment, the properties of the TL responses between our sample and TLD-100 were revealed and the glow curves of the CNTs samples were generated.

\section{Materials and Methods}

\subsection{Sample preparation}

Carbon nanotube (CNT) synthesis: CNT used in the study was synthesized by chemical vapor deposition method at a temperature of $760{ }^{\circ} \mathrm{C}$ on quartz substrate. Ferrocene was used as a source of iron catalyst and toluene was used as a carbon source in order to supply the main building element of carbon nanotubes. Toluene (laboratory reagent grade) 
was purchased from Fisher Scientific (UK) and ferrocene (98\%) was purchased from Sigma Aldrich (UK). Different weight fractions (5\%) of ferrocene in toluene were used as a feedstock which was injected using Hamilton syringe $(5.6 \mathrm{~mL} / \mathrm{h})$ into a quartz furnace via a pre-heated nozzle $\left(180{ }^{\circ} \mathrm{C}\right)$. An argon flow $(1 \mathrm{~L} / \mathrm{min})$ was also maintained into the quartz furnace throughout the synthesis procedure. The synthesis was carried out around 6 $\mathrm{h}$ producing CNT of $0.20-2.0 \mathrm{~mm}$ length in average with an average diameter of 40 to 100 $\mathrm{nm}$. CNT was synthesized on quartz tube and on the silica substrate that was placed inside the quartz tube before synthesis. CNT on silica substrate were carefully taken off with sharp knife-edge and cut into the form of carpets, which were used to make VACNT epoxy composite. CNT on quartz tube wall were collected by a scratcher and were crushed using a mortar to get this in fine powder form.

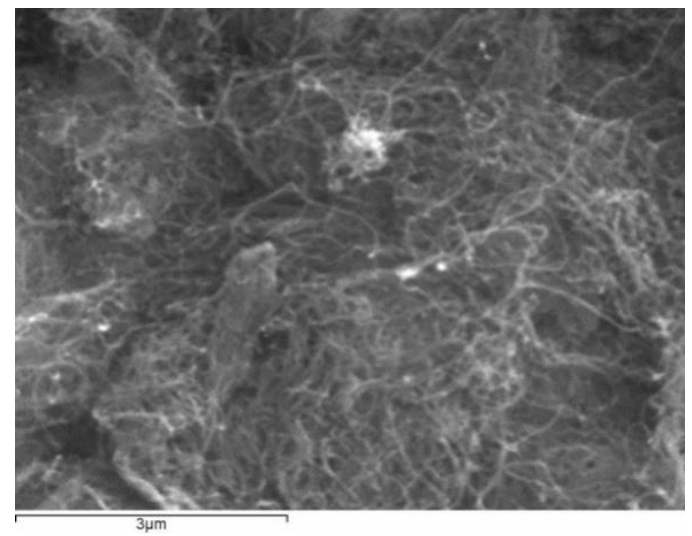

Fig. 1. Scanning electron microscope image of carbon nanotubes.

A small amount of CNT is taken in a morter which has already been washed and cleaned well by acetone. The sample is blended well in the morter for about $4 \mathrm{~h}$. Carefulness must be taken to avoid its inhalation. Five samples have been prepared in different tube, each consisting of total $50 \mathrm{~mL}$ solution. Each of the tubes is shaked well and kept at about $4 \mathrm{~h}$ for sonication in a sonicator. Five filter papers are taken to collect the dispersed CNT. Those filter papers are wetted by pouring the mixture solution of CNT and acetone on it. The solution is poured in such a way that it made a circular shape on the filter paper. Then those filter papers are kept in a hot plate at about $40{ }^{\circ} \mathrm{C}$ for some time and then those become completely dried. The circular shaped form of dispersed CNT residue on those filter papers is then ready for irradiation.

Preparation of mixed pellet $(T L D-100+C N T)$ sample: Least amount of CNT and TLD100 is taken in a morter with a total weight of the mixture is $0.055 \mathrm{~g}$ where the amount of CNT is $3 \%$ of the total weight. The mixture is blended well in the morter for about $6 \mathrm{~h}$. After blending, the mixture is separated from the morter and taken in a HYDRAULIC pressure machine at $10 \mathrm{kN}$ to form it in a pellet shape. The pellet is kept in a furnace for 2 $\mathrm{h}$ at around $300{ }^{\circ} \mathrm{C} .5 \%$ of the total weight of CNT is used in the same process to make another pellet. $7 \%$ of the total weight of CNT is used in the same process to make another 
pellet. After being kept in the furnace, all three pellets become harder for irradiation. It is important to have a minimum pellet weight of around $0.055 \mathrm{~g}$ otherwise the pellet will be tough to be formed.

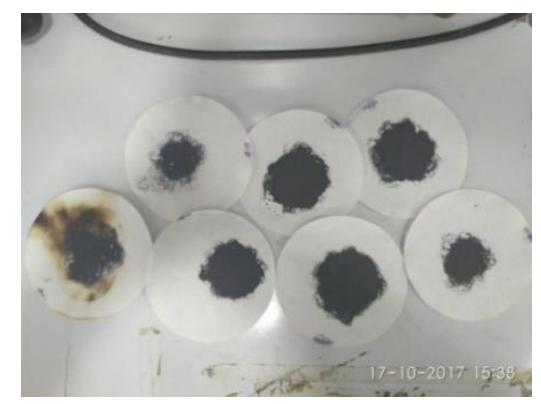

Fig. 2. Circular shaped dispersed CNT residue CNT on filter paper.

Preparation of pellet (NaI-LiF) sample: Least amount of $\mathrm{LiF}$ and $\mathrm{NaI}$ are taken in a morter so that the total weight of the mixture is $0.3 \mathrm{~g}$ where the amount of each is $50 \%$ of the total weight. The mixture is blended well in the morter for about $3 \mathrm{~h}$. After blending, the mixture is separated from the morter and taken in a HYDRAULIC pressure machine at $10 \mathrm{kN}$ to form it in a pellet shape. The pellet is kept in a furnace for $2 \mathrm{~h}$ at around $300^{\circ} \mathrm{C}$. Three pellets of the same concentration are made. After being kept in the furnace, all three pellets are harder and ready for irradiation.

\subsection{Irradiation}

Tailor-made carbon nanotubes mixed materials and various commercial TLD chips were irradiated with a $6 \mathrm{MV}$ X-ray photon beam using a Clinical Linear accelerator (VARIAN Model LINAC IX), the dose range is from 0.5 to $5 \mathrm{~Gy}$. The field size was set at $10 \mathrm{~cm} \times$ $10 \mathrm{~cm}$ and the SSD (source to surface distance) was $100 \mathrm{~cm}$ for all irradiations $\left(\mathrm{d}_{\max }=1.5\right.$ $\mathrm{cm})$. For irradiation, the samples were sandwiched between slabs of solid water phantom (standard tissue-equivalent medium) to deliver backscattering conditions. During irradiation, the dose rate was kept constant at $300 \mathrm{MU} / \mathrm{min}$ ( $1 \mathrm{MU}$ is equivalent to $1 \mathrm{cGy}$ ).

\subsection{TL measurements}

Carbonnanotubes mixed materials and standard TLD chips were read out under identical conditions using a Harshaw 3500 TL reader. The following parameters were used: preheat temperature of $50{ }^{\circ} \mathrm{C}$ for $0 \mathrm{sec}$, acquisition temperature $300{ }^{\circ} \mathrm{C}$ and heating rate at 10 ${ }^{\circ} \mathrm{C} / \mathrm{sec}$. Finally, the annealing temperature was set at $300{ }^{\circ} \mathrm{C}$ for $0 \mathrm{sec}$ to eliminate any residual signal. The measurement processes were completed under $\mathrm{N}_{2}$ gas flow, suppressing oxidation and potential thermo-luminescence. In this case, the luminescence intensity produced by heating the sample inside the TLD reader is directly proportional to the absorbed dose of radiation. 


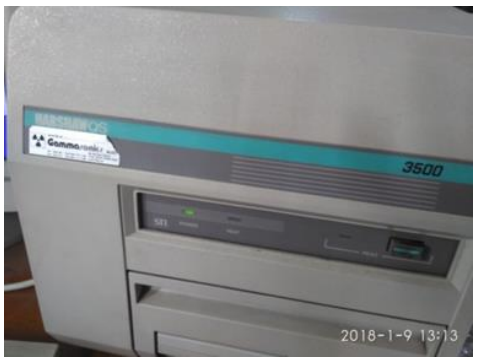

Fig. 3. Harshaw Model 3500 TLD reader.

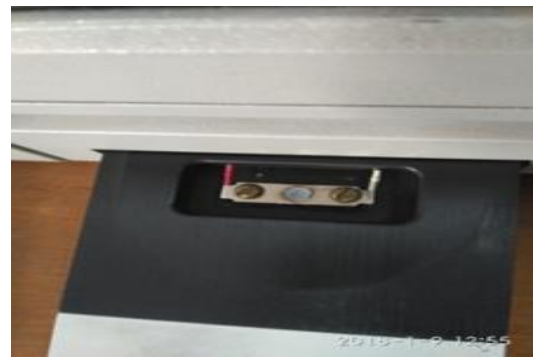

Fig. 4. Sample putting in the TLD Reader.

\section{Results and Discussion}

\subsection{Glow curves}

The glow curve is obtained by measuring the intensity of emitted light against the temperature and the period during which the temperature is increased. If the heating rate remains constant, the temperature will be proportional to the time during which heating is applied. The de-convolved peaks are numbered as a fraction of increasing trap depth. Note that the low-temperature peaks have high fading of stored signals and a very short halflife.

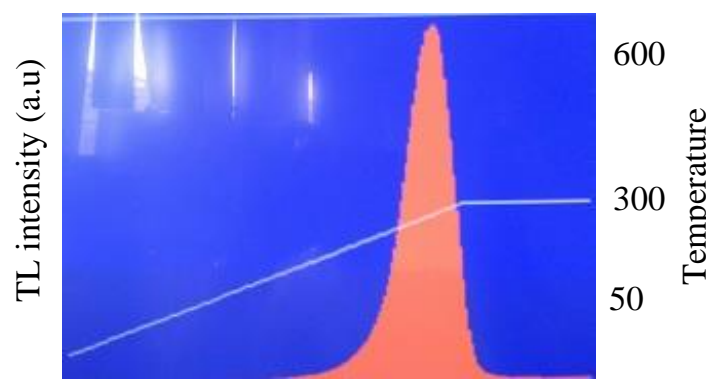

No of Channels

Fig. 5. TL glow curve of TLD 7000.

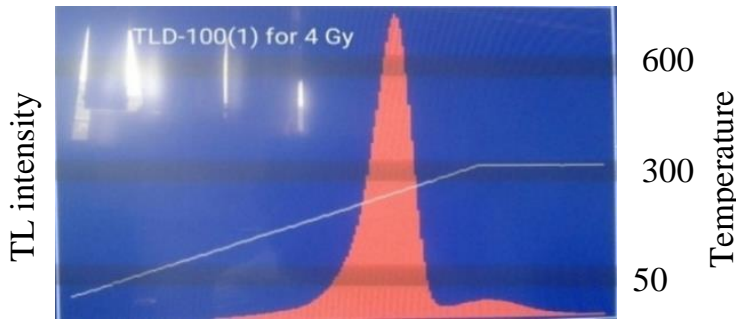

No of Channels

Fig. 6. TL glow curve of TLD100 at 4 Gy. 


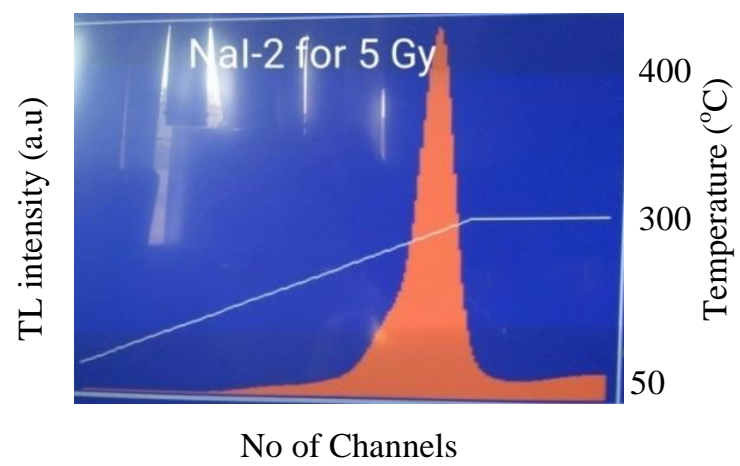

Fig. 7. TL glow curve of NaI-LiF at $5 \mathrm{~Gy}$.

The time-temperature profile (TTP) of TLD reader was set for standard TLDs that is why higher temperature is needed to release dosimetric traps. Without a higher temperature, traps will not be released. Fig. 5 shows the TL glow curve of TLD 7000 irradiated with 1 Gy. Fig. 6 shows the specific TL glow curve of TLD 100 irradiated with 4 Gy and Fig. 7 shows the TL glow curve of NaI-LiF pellets irradiated with 5 Gy of $6 \mathrm{MV}$ photon beam. At $300{ }^{\circ} \mathrm{C}$, most of the traps are started to release. For dosimetry purposes, the glow-peak of a dosimeter sample is desirable at around $200-300{ }^{\circ} \mathrm{C}$. The temperature range is important because it offers low signal fading and also represents negligible interference from the black body background signal. This type of glow curve indicates that the response is excellent.

\subsection{Photon beam response}

Dose-response linearity is one of the most desirable properties of TL material. TL response curve of TLD-100, TLD-7000, and NaI-LiF pellets was studied and shown in the Figs. 8-10, respectively.

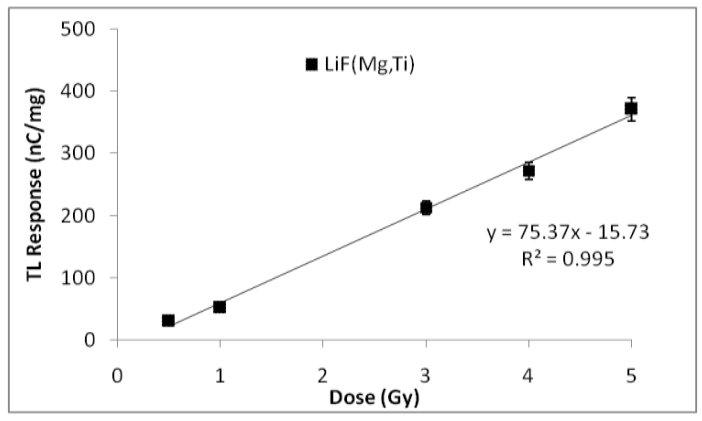

Fig. 8. TL response curve of TLD-100. 


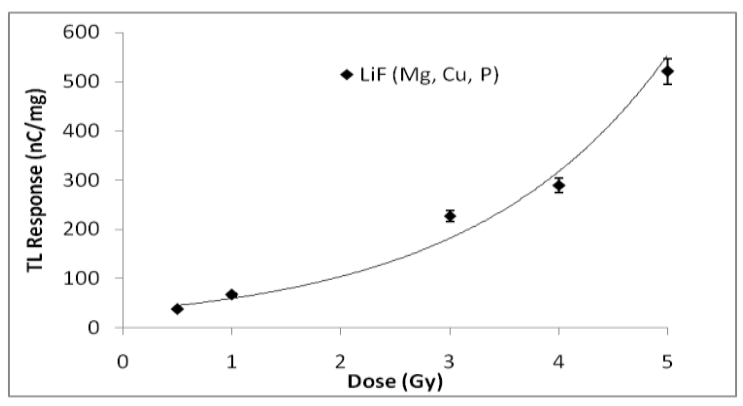

Fig. 9. TL response curve of TLD-7000.

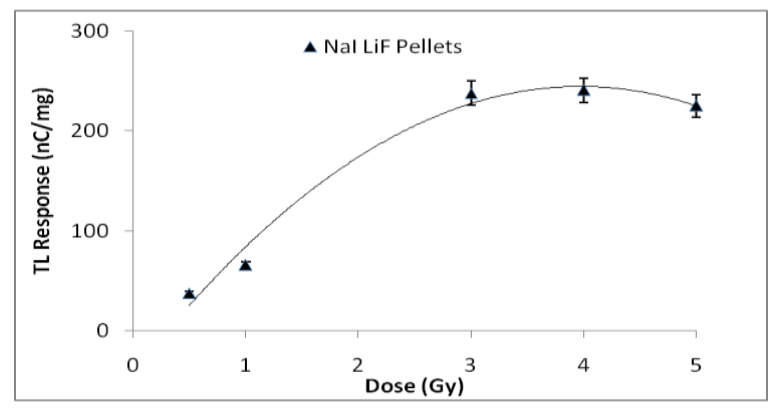

Fig. 10. TL response of NaI-LiF pellets.

From Fig. 8 it is realized that the TL response is linearly proportional to the absorbed dose and the curve follows a straight line with doses up to $5 \mathrm{~Gy}$ of the photon beam. It may occur owing to more traps at greater absorbed dose, the TL intensity is higher. That's why it doesn't need correction and calibration. From Figs. 9 and 10 it is observed that beyond dose range $2 \mathrm{~Gy}$, the TL materials provide non-linear regions in the dose-response curve. TLD-7000 provides supra-linear behavior and NaI-LiF pellets provide sub-linear behavior approaching saturation and causes under or overestimation of TL responses respectively. These two TL materials need calibration and correction from which additional errors may accumulate. Also, graphs 5 and 6 indicate that it is responsible for low doses, not for high doses.

\section{Conclusion}

TL glow peak of different CNT materials indicated that it was lying somewhere away from $300{ }^{\circ} \mathrm{C}$. TL glow peak shows a good linear relationship between TL response and absorbed dose of TLD-100 with doses up to 5 Gy of the photon beam.TLD-7000 and NaI$\mathrm{LiF}$ pellets show supra-linear and sub-linear behavior beyond $2 \mathrm{~Gy}$. The general response of the CNT materials indicates considerable potential for the raw CNT media to be used in radiotherapy dosimetric applications. Exhibition of dosimetric properties of CNTs lays 
down a promising avenue to pursue further research with this material. This research will be useful for establishing the CNTs as TL material for radiation dosimetry.

\section{References}

1. J. Cameron, Environ. Health Perspect. 91, 45 (1991). https://doi.org/10.1289/ehp.919145

2. S. Tim and P. Keith, Phil. Photography 2, 153 (2011). https://doi.org/10.1386/pop.2.1.153 7

3. R. Chen and V. Pagonis, Radiation Measurements 71, 8 (2014). https://doi.org/10.1016/j.radmeas.2013.12.011

4. Y. S. Horowitz, Radiat. Measurements 71,2 (2014). https://doi.org/10.1016/j.radmeas.2014.01.002

5. K. M. M. Hasan, F. Rahman, A. K. M. M. Rahman, M. S. Rahman, M. M. M. Siraz, S. Pervin, S. Banik, and S. Yeasmin - International Conference on Electronics and ICT (2018).

6. NE Technology Ltd, Operators Manual for Solaro TLD Reader, ver. 2, Reading (UK, 1996).

7. S. Hashim, S. Al-Ahbabi, D. A Bradley, M. Webb, C. Jeynes, A. T. Ramli, and H. Wagiran, Appl. Radiat. Isot. 67, 423 (2009). https://doi.org/10.1016/j.apradiso.2008.06.030

8. F. M. Khan, The Physics of Radiation Therapy, $3^{\text {rd }}$ Edition (Lippincott Williams \& Wilkins, New York, USA, 2003).

9. H. Bicron, Model 3500 Manual TLD Reader (Ohio: Saint-Gobain/Norton Industrial Ceramics Corporation, 1993). 